\title{
A Case of Fifteen Simultaneous Rectal Neuroendocrine Tumors and Endocrine Cell Micronests Resected by Both Endoscopic Treatments and Surgery
}

\author{
Hikaru Hashimoto ${ }^{a}$ Naohisa Yoshida ${ }^{a}$ Ken Inoue ${ }^{a}$ Reo Kobayashi ${ }^{a}$ \\ Yuri Tomita ${ }^{a}$ Satoshi Sugino ${ }^{a}$ Osamu Dohi ${ }^{a}$ Ryohei Hirose ${ }^{a}$ \\ ${\text { Yutaka Inadab }{ }^{\mathrm{b}} \text { Takaaki Murakami }{ }^{\mathrm{c}} \text { Yukiko Morinagad }}^{d}$ \\ Mitsuo Kishimoto ${ }^{\mathrm{e}}$ Yoshito Itoh $^{\mathrm{a}}$ \\ aDepartment of Molecular Gastroenterology and Hepatology, Graduate School of \\ Medical Science, Kyoto Prefectural University of Medicine, Kyoto, Japan; 'bepartment \\ of Gastroenterology, Kyoto First Red Cross Hospital, Kyoto, Japan; 'Department of \\ Gastroenterology, Aiseikai Yamashina Hospital, Kyoto, Japan; dDepartment of Surgical \\ Pathology, Kyoto Prefectural University of Medicine, Kyoto, Japan; ${ }^{\text {eDepartment of }}$ \\ Pathology, Kyoto City Hospital, Kyoto, Japan
}

\section{Keywords}

Rectal neuroendocrine tumor · Endoscopic submucosal dissection · Endocrine cell micronests

\begin{abstract}
Case Report: A 65-year-old man without any symptoms received colonoscopy for cancer screening and underwent cold snare polypectomy (CSP) for a 3-mm rectal lesion at a local clinic. A histopathological examination revealed neuroendocrine tumor (NET) G1 with a positive margin. The patient was referred to our hospital for further treatment. Then, the post-CSP scar was removed by endoscopic submucosal dissection (ESD), with a sufficient endoscopically normal margin. Histopathology showed 4 NETs and endocrine cell micronests (ECMs) distant from the post-CSP scar, with a positive lateral margin. We considered that the possibility of other NETs was high. Additional surgery was performed. After a histopathological examination, 11 NETs and ECMs were found in the whole rectum, without lymph node metastasis. The patient had no recurrence at 24 months after surgery. In the past 10 years, we have experienced 4 cases (including the present case) of multiple rectal NETs among 56 cases of rectal NETs of $\leq 10 \mathrm{~mm}$ (7.1\%). None of our 4 cases showed any recurrence (follow-up period: 12-32 months).Conclusions: We herein report a case involving a patient with 15 rectal NETs and
\end{abstract}


ECMs. We reviewed our experience with multiple rectal NETs, and the rate of multiple rectal NETs was $7.1 \%$. Endoscopists should consider that multiple lesions may be present in cases of rectal NET and be aware that some cannot be detected endoscopically.

(C) 2022 The Author(s).

Published by S. Karger AG, Basel

\section{Introduction}

Neuroendocrine tumors (NETs) were divided into highly differentiated NETs and poorly differentiated neuroendocrine carcinomas (NECs) in the WHO classification [1]. Most gastrointestinal tract NETs are single and are located at the lower rectum. Risk factors for lymph node metastasis (LNM), including lymphatic-venous invasion, grade G2 or G3, and invasion to the proper muscle layer are regularly considered for rectal NETs of $\geq 10 \mathrm{~mm}$. According to the risk factors, surgery is recommended for some cases [1,2]. Multiple rectal NETs are sometimes reported, but their characteristics, including LNM, remain unclear [3]. NETs rarely include endocrine cell micronests (ECMs), which are reported as clusters of endocrine cells of $<0.5 \mathrm{~mm}$ in size, and the details of ECMs are also unclear [4]. In this case report, we presented the case of a patient with 15 rectal NETs and ECMs.

\section{Case Report}

The patient was a 65-year-old man without any symptoms who had undergone colonoscopy for cancer screening at a local clinic. Colonoscopy showed a 3-mm yellowish polypoid lesion on the lower rectum, and cold snare polypectomy (CSP) was performed (Fig. 1a). The histopathological diagnosis was NET G1 with positive horizontal and vertical margins (Fig. 1b,

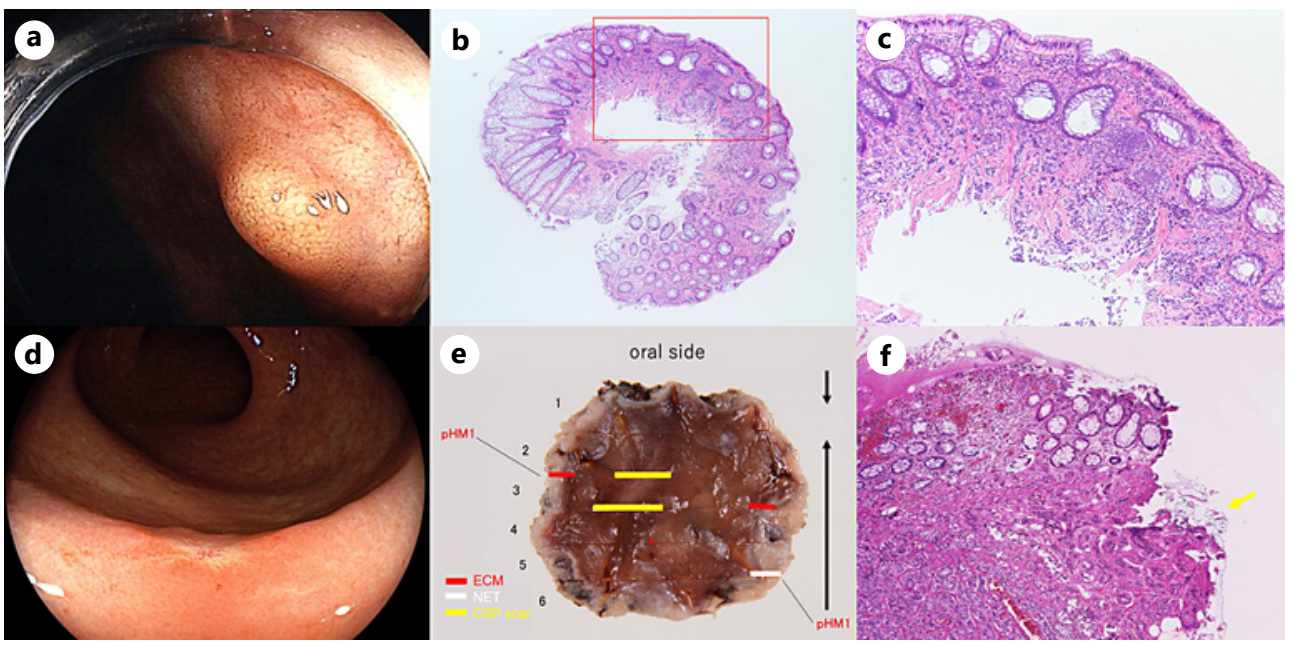

Fig. 1. A rectal NET and ECMs resected with initial CSP and following ESD. a A yellow-colored polypoid tumor of $3 \mathrm{~mm}$ in size on the lower rectum. b A low-power histopathological specimen obtained by CSP. The red square is magnified in (c) $(\mathrm{H} \& \mathrm{E} . \times 12.5)$. c A histopathological examination showed an NET with positive vertical margins (H\&E. $\times 100)$. d A flat scar without submucosal elevation due to cold snare polypectomy. e A histopathological examination showed three ECMs and one NET located away from the ESD scar (HM, horizontal margin). $\mathbf{f}$ An NET of $2.8 \mathrm{~mm}$ in size was detected, and the horizontal margin was positive (yellow arrow). It was mainly located in the submucosal layer (H\&E. $\times 100)$. 


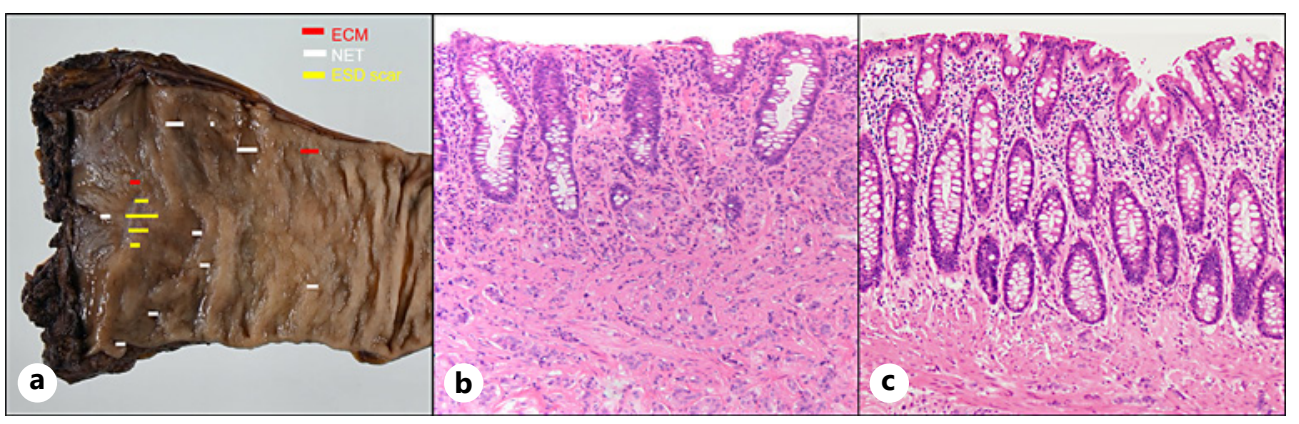

Fig. 2. Eleven NETs and ECMs resected with surgery. a A resected specimen. Eleven lesions of NETs and ECMs were found. $\mathbf{b}$ In a histopathological examination, in addition to the ESD scar, NET was detected from the mucosa to the submucosal layer (H\&E. $\times 200)$. c Histopathological examinations showed that in addition to the ESD scar, ECMs were present, mainly in the mucosa and partially in the submucosa (H\&E. $\times 200$ ).

c). He was referred to our hospital for further treatment. He had no history of specific diseases, including ulcerative colitis, gastric NET, and other NETs. None of his relatives had been diagnosed with NETs. Blood examinations showed no abnormalities. Hormones related to NET (e.g., gastrin) were not examined because he had no symptoms. Abdominal computed tomography (CT) showed no evidence of distant metastasis and other NETs. Colonoscopy showed only a post-CSP scar without submucosal elevation, or any residual and other NET lesions (Fig. 1d). For a further histopathological evaluation, the post-CSP scar was removed by endoscopic submucosal resection (ESD). With ESD, a sufficient horizontal margin of 5-10 mm from the scar was obtained. The histopathological examination showed 4 NETs and ECMs distant from the post-CSP scar, with a positive margin (Fig. 1e, f). Immunohistochemistry showed chromogranin A, synaptophysin, and CD56 positivity. Additionally, lymphatic invasion was detected with D2-40. Thus, we thought the possibility of a residual NET, multiple other rectal NETs, and lymph node metastasis of NET. According to these possibilities, we treated the patient surgically. Robotic-assisted laparoscopic intersphincteric resection and D2 lymph node dissection was performed due to the positive margin and lymphatic invasion (Fig. 2a). A histopathological examination showed 11 NETs and ECMs in the whole rectum. ECMs were found in the mucosa and submucosa (Fig. 2b, c). There were no findings of chronic inflammation in the background mucosa. The vertical and horizontal margins were negative, and no LNM was observed. The patient, who received annual colonoscopy and CT, showed no recurrence within 24 months after the surgery.

\section{Discussion}

Due to the increased performance of colonoscopy, a decade-long incremental trend has been observed for both NET and NEC [1]. Endoscopic submucosal resection with ligation, ESD, and precutting EMR have been reported to be useful for rectal NETs [5, 6]. However, small NETs are sometimes resected by CSP, although the indication of CSP is for benign lesions of $<10 \mathrm{~mm}$ in size. It results in an insufficient histopathological evaluation and positive margins. To prevent this kind of cases, endoscopists should decide an appropriate therapeutic approach according to the endoscopic diagnosis of each lesion, using magnified endoscopy if possible. Rectal submucosal tumors should not be resected by CSP because they may be NETs.

Regarding LNM according to tumor size, Konishi et al. [2] reported that LNM occurred in $7 \%, 30 \%$, and $58 \%$ of cases with tumors of $1-10 \mathrm{~mm}, 11-20 \mathrm{~mm}$, and $\geq 21 \mathrm{~mm}$, respectively. We previously reported that the LNM rate among 132 rectal NETs $\leq 10 \mathrm{~mm}$ was $3.0 \%$ (95\% CI: 


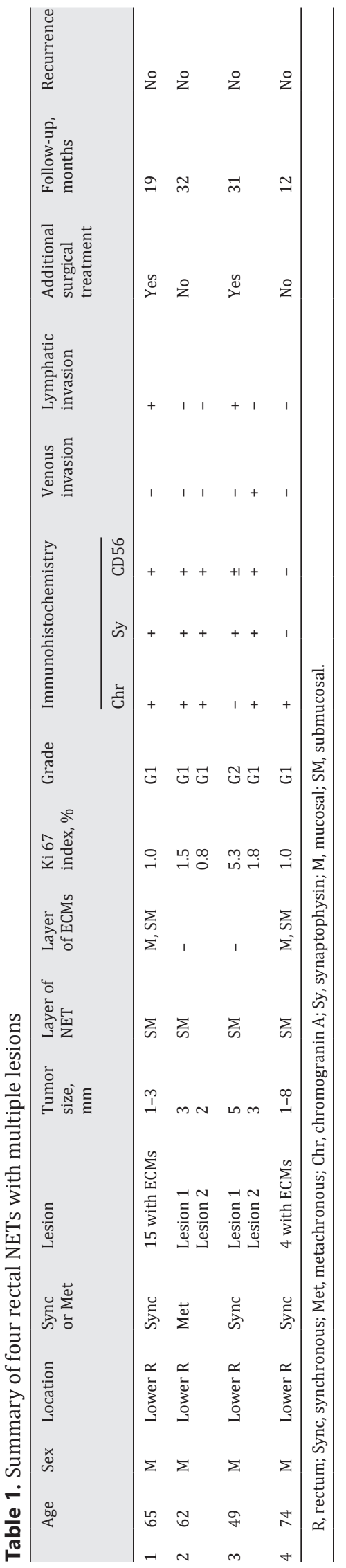




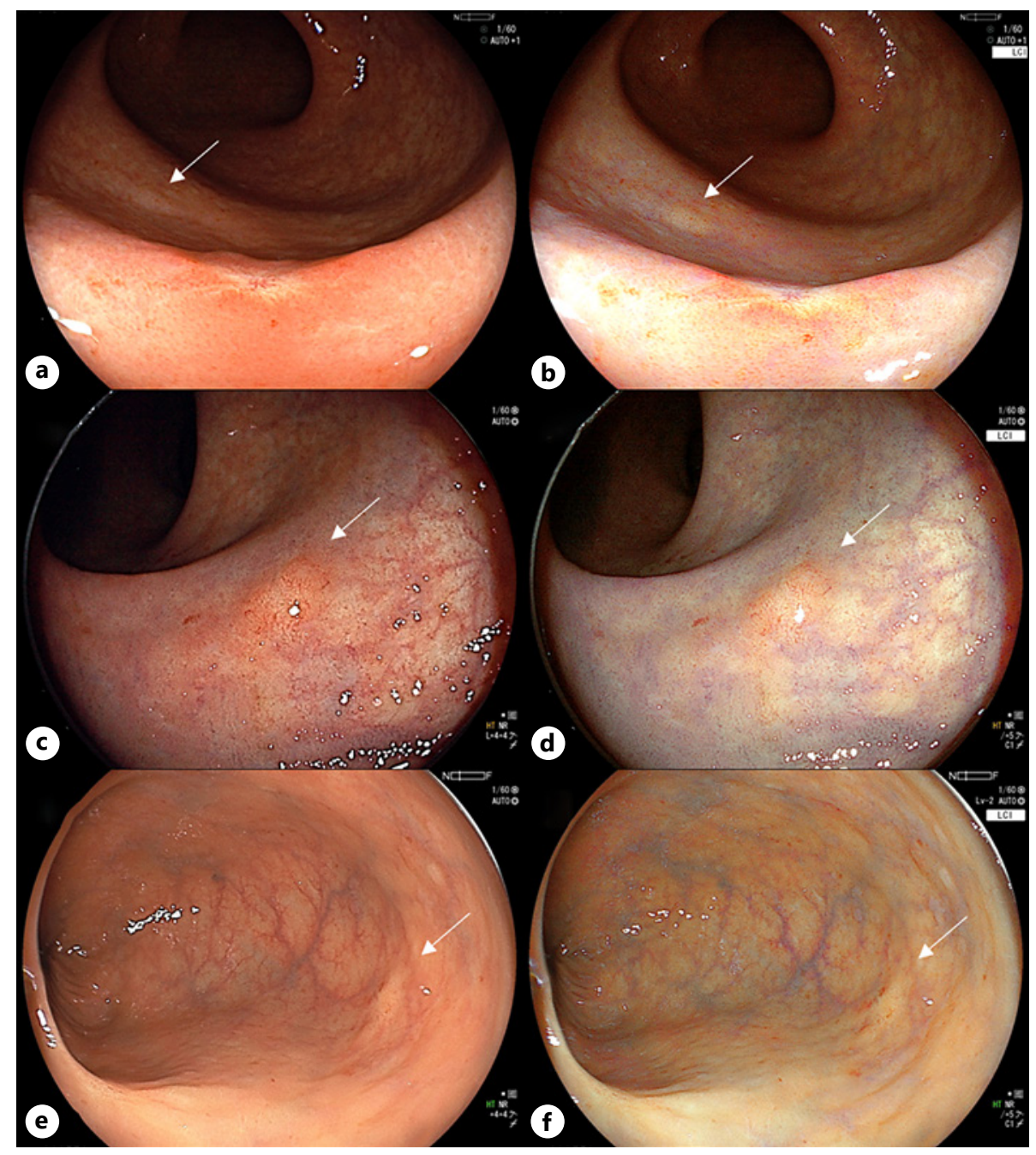

Fig. 3. Small NETs observed with WLI and LCI. a A residual lesion at the oral side of the post-CSP scar of case 1 in Table 1 with WLI (white arrow). b LCI made the lesions more detectable (white arrow). c Case 3 in Table 1 with WLI (white arrow). d LCI made the lesion more detectable (white arrow). e Case 4 in Table 1 with WLI (white arrow). f LCI made the lesion more detectable (white arrow).

0.97\%-7.7\%), while lesions of $\leq 6 \mathrm{~mm}$ showed no lymph node metastasis (95\% CI: 0-4.7\%) [6]. A study that included 90 rectal NETs of $\leq 13 \mathrm{~mm}$ resected endoscopically reported an excellent long-term prognosis, without clinical recurrence after endoscopic resection (median follow-up time: 67.5 months), although 42 lesions (46.7\%) were positive for lymphatic invasion [7]. However, even for lesions of $\leq 10 \mathrm{~mm}$, a histopathological examination of endoscopically resected specimens sometimes reveals invasion of the proper muscle layer, positive tumor margin, high grade, or lymphatic-venous invasion, and additional surgery is considered. In our case, the positive margin and lymphatic invasion were detected histopathologically, and surgery was performed. However, the possibility of LNM for lesions of $\leq 10 \mathrm{~mm}$ remains unclear, and it is difficult for clinicians to decide the therapeutic methods for such lesions. A Japanese largescale prospective study on rectal NET is now ongoing and will clarify this issue.

Most rectal NETs show a single lesion; however, there are some reports about multiple lesions [3, 8-10]. It is not well understood why multiple lesions develop. However, a report on multiple rectal NETs in monozygotic twins suggested that the origin was related to their 
identical innate genetic background, and not environmental factors or acquired genomic alterations [9]. Previous reports showed that the rate of multiple lesions was $1.4-4.5 \%$ [3, 10]. Among 56 cases of NETs of $<10 \mathrm{~mm}$ in our hospital from 2010 to 2020 , we identified 4 cases of multiple rectal NETs (7.1\%) (Table 1). Two cases (case 1: the current case and case 4) had $>3$ NET lesions with ECMs, and no LNM was noted. While the relationship between the presence of multiple NETs with ECMs and the prognosis are unclear, none of our 4 cases showed any recurrence (follow-up period: 12-32 months). Our cases suggest that patients with multiple rectal NETs do not have a bad prognosis; however, a further analysis should be performed.

Endoscopists should consider the possibility of multiple NETs and be careful for other yellowish areas in the rectum before and after endoscopic treatment of rectal NET. However, ECM and small NETs are difficult to detect endoscopically with white light imaging (WLI). Linked color imaging (LCI; Fujifilm Co., Tokyo, Japan) is a kind of narrowbanding image for detecting gastrointestinal neoplastic lesions. LCI makes lesions more reddish and makes white area more whitish [11-13]. In our multiple cases in Table 1, small NETs were emphasized, appearing whiter with LCI in comparison with WLI (Fig. 3). The retrospective evaluation of endoscopic figures in case 1 showed that both the residual NET lesion and a part of the other NET lesions could be clearly detected with LCI. Further studies should be performed to investigate the accuracy of LCI in the detection of small NET lesions.

It has not been confirmed that ECM is a malignant lesion (pre-NET lesion) $[4,8]$. In the current report, 2 cases with $\geq 4$ lesions had ECM (Table 1), and there was no LNM. Similar to our 2 cases, some studies reported that cases with ECM did not have metastasis or recurrence $[4,8]$. A further large-scale study including genetic analysis should be performed to clarify the characteristics of ECM.

In conclusion, we described the case a patient with 15 simultaneous rectal NETs and ECMs. We also reviewed our multiple rectal NETs, and the rate of multiple rectal NETs was $7.1 \%$. When endoscopists encounter rectal NET, they should consider the possibility of multiple rectal NET lesions.

\section{Acknowledgments}

We thank Dr. Hitoshi Okano and all members of the Department of Molecular Gastroenterology and Hepatology, Kyoto Prefectural University of Medicine, for helping with this report.

\section{Statement of Ethics}

We obtained written informed consent from the patient for publication of this case report and any accompanying images. This case report was performed with the approval of the Ethics Committee of Kyoto Prefectural University of Medicine (ERB-C-1600) and was in accordance with the World Medical Association Declaration of Helsinki.

\section{Conflict of Interest Statement}

All of authors declare no conflicts of interest in association with the present study.

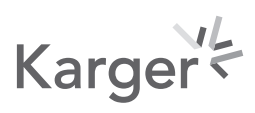


Hashimoto et al.: Multiple Rectal Neuroendocrine Tumors

\section{Funding Sources}

This manuscript did not receive any funding.

\section{Author Contributions}

Hasimoto H. and Yoshida N. designed the study, performed endoscopic examination and analysis, and prepared the manuscript; Tomita Y., Sugino S., and Inoue K. helped endoscopic examination; Morinaga Y. and Kishimoto M. did histological assessments; Itoh Y. arranged and reviewed the manuscript.

\section{Data Availability Statement}

All data generated or analyzed during this study are included in this article. Further enquiries can be directed to the corresponding author.

\section{References}

1 Ringi G, Komminoth P, Scoazec JY, Shia J. Colorectal neuroendocrine neoplasms: WHO Classification of Tumours Editorial Board, editor. WHO classification of tumours. Digestive system tumours. 5th ed. Lyon: International Agency for Research on Cancer; 2019. p. 188-92.

2 Konishi T, Watanabe T, Kishimoto J, Kotake K, Muto T, Nagawa H. Prognosis and risk factors of metastasis in colorectal carcinoids: results of a nationwide registry over 15 years. Gut. 2007;56:863-8.

3 Sasou S, Suto T, Satoh T, Tamura G, Kudara N. Multiple carcinoid tumors of the rectum: report of two cases suggesting the origin of carcinoid tumors. Pathol Int. 2012;62:699-703.

4 Abe H, Kubota K, Oka T, Kobayashi T, Makuuchi M. A rare case of multiple carcinoids and endocrine cell micronests in a patient with chronic duodenitis. Cancer. 2000;89:963-9.

5 Niimi K, Goto O, Fujishiro M, Kodashima S, Ono S, Mochizuki S, et al. Endoscopic mucosal resection with a ligation device or endoscopic submucosal dissection for rectal carcinoid tumors: an analysis of 24 consecutive cases. Dig Endosc. 2012;24:443-7.

6 Inada Y, Yoshida N, Fukumoto K, Hirose R, Inoue K, Dohi O, et al. Risk of lymph node metastasis after endoscopic treatment for rectal NETs 10 mm or less. Int J Colorectal Dis. 2021;36:559-67.

7 Sekiguchi M, Sekine S, Sakamoto T, Otake Y, Nakajima T, Matsuda T, et al. Excellent prognosis following endoscopic resection of patients with rectal neuroendocrine tumors despite the frequent presence of lymphovascular invasion. J Gastroenterol. 2015;50:1184-9.

8 Suzuki S, Kawakami H, Miike T, Yamamoto S, Abe H, Shimoda K, et al. Single rectal neuroendocrine tumor associated with multiple endocrine cell micronests. Intern Med. 2020;59:619-23.

9 Doi M, Ikawa 0, Taniguchi H, Kawamura T, Katsura K. Multiple rectal carcinoid tumors in monozygotic twins. Clin J Gastroenterol. 2016;9:215-21.

10 Soga J. Carcinoid of the rectum: tumors of the carcinoid family: urgut endocrinomas. Acta Med Biol. 1982;29: 157-201.

11 Shinozaki S, Kobayashi Y, Hayashi Y, Sakamoto H, Sunada K, Lefor AK, et al. Colon polyp detection using linked color imaging compared to white light imaging: systematic review and meta-analysis. Dig Endosc. 2020;32: 874-81.

12 Ono S, Kawada K, Dohi O, Kitamura S, Koike T, Hori S, et al. Linked color imaging focused on neoplasm detection in the upper gastrointestinal tract: a randomized trial. Ann Intern Med. 2021;174:18-24.

13 Yoshida N, Hisabe T, Ikematsu H, Ishihara H, Terasawa M, Inaba A, et al. Comparison between linked color imaging and blue laser imaging for improving the visibility of flat colorectal polyps: a multicenter pilot study. Dig Dis Sci. 2020;65:2054-62. 